\title{
An ultra-high sensitivity temperature sensor based on surface-plasmon polariton in metal-insulator-metal waveguide and a dynamic cavity
}

\author{
Zhiliang Chen $^{1, a}$, Zhengbiao Ouyang ${ }^{1, b}$, Qiang Liu ${ }^{1, c}$, Yaoxian Zheng ${ }^{1, d}$ \\ College of Electronic Science and Technology, Shenzhen University, Shenzhen, \\ Guangdong 518060, China \\ achenzhiliang@email.szu.edu.cn, bzbouyang@szu.edu.cn, corresponding \\ author, 'gnaiqliu@hotmail.com, djewel5282@163.com
}

Keywords: Surface plasmon; Temperature; sensor

\begin{abstract}
A temperature sensor based on surface-plasmon polariton in metal-insulator-metal waveguide and a half-open dynamic cavity with a movable silver granule driven by thermal expansion from a small cube of ethanol is proposed. Numerical simulations through finite-element method demonstrated that the temperature sensor can have a resolution as extremely fine as $0.412 \times 10^{-9}{ }^{\circ} \mathrm{C}$, The proposed design may find applications in micro-sensor chips for high-sensitivity measuring of temperature varying slowly in mini regions.
\end{abstract}

\section{Introduction}

Temperature sensors have shown wide applications, e.g., thermometers in daily life, large temperature-controlled equipment and integrated-circuit temperature sensors [1]. To pursue high sensitivity, the optical fiber temperature sensor has attracted great attention [2-5]. For example, a liquid-crystal photonic-crystal-fiber temperature sensor with a high sensitivity of $105 \mathrm{~nm} /{ }^{\circ} \mathrm{C}$ was proposed by Wang et al. [4]. However, the above mentioned designs are not cost-effective in micro-scale. It is also noted that A micro-nanostructure temperature sensor based on surface plasmon, but its sensitivity is only $0.65 \mathrm{~nm} /{ }^{\circ} \mathrm{C}$ was proposed by $\mathrm{Wu}$ et al. [6]. On the other hand, temperature sensors based on surface plasmons have become hot spots recently for their potential application in miniaturizations [7-8]. Most of the surface-plasmon temperature sensors proposed in the past are based on optical fibers, which are not preferred for micro integrated circuits [7-8].

In this paper, An ultra-high sensitivity temperature sensors based on SPP in metal-insulator-metal (MIM) waveguide and a half-open dynamic cavity with a movable silver granule driven by thermal expansion from a small cube of ethanol are presented. It is demonstrated that the temperature sensor can have a resolution as extremely fine as $0.412 \times 10^{-9}{ }^{\circ} \mathrm{C}$. Such a high resolution sensor may find application for measuring extremely week signals, e.g. remote star radiations, gravitational wave signals, and the energy of a single particle.

\section{Structure and materials of the temperature sensor}

The basic part of the proposed SPP temperature sensor is schematically shown in Fig. 1(a), which consists of a cube of ethanol, a horizontal waveguide and a dynamic cavity with a movable silver granule. A cube filled with ethanol is placed outside the MIM structure which not only makes the structure of the sensor more compact and has short response time but also improves the sensitivity of the temperature sensor. The sensor is for high-sensitivity measuring of temperature, in which the temperature variation can be assumed to vary very slowly. So, the silver granule moves very slowly and the friction of silver granule inside the waveguide can be neglected. The movement of the silver granule will changes the length of the dynamic cavity, which will in turn lead to the shift of transmission spectrum or the intensity change of the output light. 

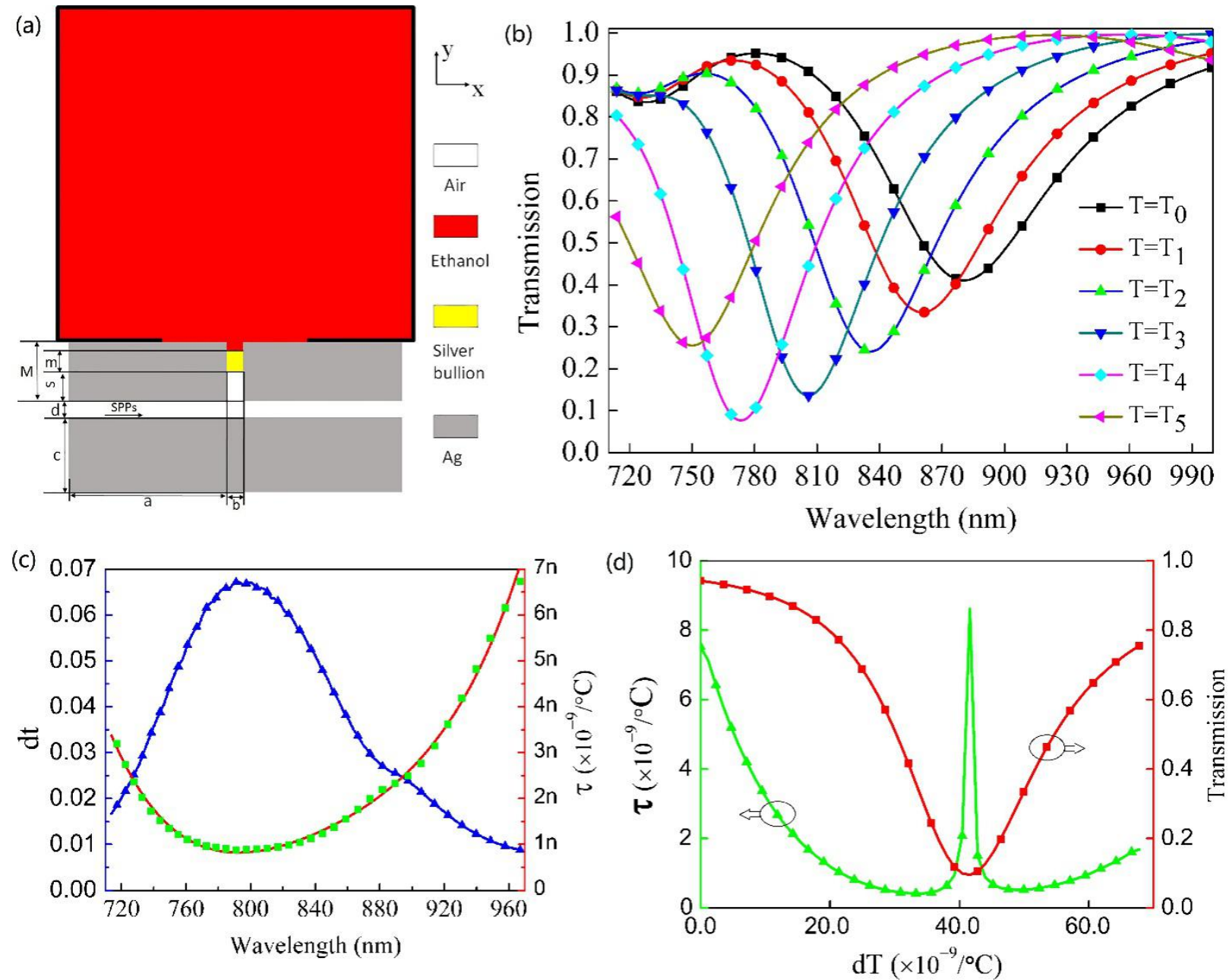

Fig. 1. (a) Basic part of the temperature sensor. (b) Transmission spectra of the sensor at different temperatures. (c) Transmission variation versus wavelength. (d) Transmission at the wavelength 792 $\mathrm{nm}$ (red curve) and the resolutions (green curve) versus temperature variation. For the temperature variations of $0{ }^{\circ} \mathrm{C}, 2.0 \times 10^{-8}{ }^{\circ} \mathrm{C}, 4.0 \times 10^{-8}{ }^{\circ} \mathrm{C}, 6.0 \times 10^{-8}{ }^{\circ} \mathrm{C}$, the values of $s$ are $160 \mathrm{~nm}, 133 \mathrm{~nm}, 106$ $\mathrm{nm}, 79.9 \mathrm{~nm}$ at the corresponding temperature, respectively.

The permittivity of silver can be described by Drude-Lorentzian model [9]. In order to have a distinct movement of the movable silver granule, the volume-expansion coefficient of the material in the cube should be large enough ethanol is ideal materials considering environmental protection. The volume-expansion coefficient of ethanol and the linear coefficient of expansion for silver are $\alpha_{\text {ethanol }}=1.1 \times 10^{-3} /{ }^{\circ} \mathrm{C}$ and $\alpha_{A g}=19.7 \times 10^{-6} /{ }^{\circ} \mathrm{C}$, while the upper and under surfaces are sealed by dielectric material, e.g., silicon and silicon dioxide. The thermal-expansion coefficient of the cube wall (the black areas around ethanol in Fig. 1(a)) should be low and the heat-conduction coefficient of the cube wall should be as high as possible. The best material is silicon carbide because its thermal-expansion and heat-conduction coefficients are better than that of other materials. The parameters of the designed structure are shown in Table 1.

Table 1: Summary of structural parameters

\begin{tabular}{lllllllll}
\hline Parameter & $\mathrm{b} / \mathrm{nm}$ & $\mathrm{H} / \mathrm{mm}$ & $\mathrm{M} / \mathrm{nm}$ & $\mathrm{m} / \mathrm{nm}$ & $\mathrm{a} / \mathrm{nm}$ & $\mathrm{c} / \mathrm{nm}$ & $\mathrm{d} / \mathrm{nm}$ & $\mathrm{h} 1 / \mathrm{nm}$ \\
\hline Value & 35 & 1.612 & 300 & 125 & 400 & 200 & 50 & 100 \\
\hline
\end{tabular}

\section{Simulation result and discussion}

We can calculate the displacement of the silver granule which is a function of temperature:

$$
\sigma=b^{-1} h_{1}^{-1} \alpha_{\text {ethanol }} V=b^{-1} h_{1}^{-1} \alpha_{\text {ethanol }} H^{3}
$$

where $V$ is the volume of ethanol cavity, $H$ is the length of ethanol cube, $b$ is the width of the 
dynamic cavity which is also the width of the movable silver granule, and $h_{1}$ is the thickness of the silver granule. According to the parameters of the structure and Eq. (1), the value of $\sigma$ is $1.316 \times 10^{9} \mathrm{~nm} /{ }^{\circ} \mathrm{C}$.

The temperature resolution of the sensor based on single-wavelength response of light can be calculated by

$$
\tau=\Delta t|d T / d t|_{\min }=\Delta t /|d t / d T|_{\max }
$$

where $t$ and $T$ denote the transmission and temperature respectively, while $\Delta t$ denotes the minimum transmission difference detectable by the opto-electronic detector.

Generally, different wavelength of light corresponds to different transmission of light through the system, so optimum operating wavelength is to be determined for obtaining optimum temperature resolution of the sensor. Let us consider the transmission spectra for different temperature. From these spectra, we can calculate the absolute value of transmittance variation as follows.

$$
d t(\lambda)=\frac{1}{N} \sum_{i=1}^{N-1}\left|t_{i+1}(\lambda)-t_{i}(\lambda)\right|
$$

where $t_{i}(\lambda)$ is the transmission at the temperature $T_{i}$ and wavelength $\lambda$. As the temperature changes, the silver granule would move, so that $T_{i}$ corresponds to a certain position of the granule. Therefore, we can first plot out the transmission-variation spectrum according to Eq. (3). Then the wavelength showing maximum transmission variation in the spectrum is the optimum operating wavelength to be found.

Finite-element method is applied to numerically investigate the sensor. In simulation the initial temperature is $20^{\circ} \mathrm{C}$. Considering that the freezing and boiling points of ethanol are $-114{ }^{\circ} \mathrm{C}$ and $78.4{ }^{\circ} \mathrm{C}$, respectively, the temperature sensor has to operate within this range. A light source with broad band is applied to study the spectrum response, as shown in Fig. 1(b). The variation of temperature is $d T=1.189 \times 10^{-8}{ }^{\circ} \mathrm{C}, T_{0}$ is the initial temperature for the initial value of $s=s_{i}=160$ $\mathrm{nm}, T_{1}=T_{0}+d T$ for $s_{i}=144.3 \mathrm{~nm}, T_{2}=T_{0}+2 d T$ for $s_{i}=128.6 \mathrm{~nm}, T_{3}=T_{0}+3 d T$ for $s_{i}=112.9 \mathrm{~nm}$, $T_{4}=T_{0}+4 d T$ for $s_{i}=97.2 \mathrm{~nm}$, and $T_{5}=T_{0}+5 d T$ for $s_{i}=81.5 \mathrm{~nm}$. It shows that with the decrease of $s$, the transmission spectrum of the dynamic cavity shifts gradually towards the long-wavelength region due to the volume expansion of ethanol caused by the rising of temperature. The transmission characteristics of the MIM waveguide can be explained by the scattering matrix theory [9].

We first calculate the transmission-variation spectrum and the corresponding resolution spectrum, as shown in Fig. 1(c). The blue curve marked by triangles is the transmission-variation spectrum calculated by Eq. (3) and the square green point are the original data calculated by Eq. (2), while the red curve is the fitting line. From Fig. 1(c) we can find easily that there is a maximum transmission variation and smallest detectable temperature or finest resolution at the wavelength of $792 \mathrm{~nm}$, so that this wavelength is the optimum operating wavelength to be find, while the corresponding resolution is the optimum temperature resolution which is better than $0.990 \times 10^{-9}{ }^{\circ} \mathrm{C}$ by considering that the resolution $\Delta \mathrm{t}$ in measuring transmittance can be easily made better than $2 \%$.

We also investigated the transmission for the wavelength of $792 \mathrm{~nm}$ in different temperatures. The result is shown by the red curve in Fig. 1(d). From the red curve, we can calculate the differential $d t / d T\left(1 /{ }^{\circ} \mathrm{C}\right)$, and then the resolution can be calculated by Eq.(2), as shown by the green curve in Fig. 1(d). From Fig. 1(d), the finest resolution is found as $0.412 \times 10^{-9}{ }^{\circ} \mathrm{C}$ at $s=116 \mathrm{~nm}$. In practical applications such as fixed temperature measurements, the initial position of the silver granule is chosen to be at $s=116 \mathrm{~nm}$, so that the sensor can have the finest resolution, which is better than $0.412 \times 10^{-9}{ }^{\circ} \mathrm{C}$. This resolution of the sensor is finer than that of the sensors reported in Refs. [4, 6] by $10^{4}$ to $10^{6}$ times. From Fig. 1(d), we can see that the finest resolution, or the minimum value of the resolution is near the minimum transmission point, but not at that point. This is understandable as the resolution is related directly to the changing ratio of transmission while at the minimum transmission point the changing ratio is zero. 


\section{Conclusion}

An ultra-high sensitivity temperature sensors based on SPP in MIM waveguide and a half-open dynamic cavity with a movable silver granule driven by thermal expansion from a small cube of ethanol has been proposed. It has been demonstrated that the finest resolution of the sensor can reach $0.412 \times 10^{-9}{ }^{\circ} \mathrm{C}$. Furthermore, the resolution of the sensor is greatly finer than that of temperature sensors reported previously by $10^{4}$ to $10^{6}$ times. The proposed sensor may find applications in micro-sensor chips for measuring extremely week signals, e.g. remote star radiations, gravitational wave signals, and the energy of a single particle.

\section{Acknowledgments}

This work is supported by the NSFC (Grant No.: 61275043, 60877034, 61307048, 61171006), the Shenzhen Science Bureau (Grant No. 200805, CXB201105050064A, JCYJ20120613115000529).

\section{References}

[1] P. Chen, C. C. Chen, C. C. Tsai, and W. F. Lu, "A Time-to-Digital-Converter-Based CMOS Smart Temperature Sensor," IEEE J. Solid-St. Circ. 40 (2005) 1642-1648.

[2] A. M. Ríos, G. A. Sanchez, R. S. Aguilar, A. A. C. Guzman, D. T. Acosta and C. A. C. Arriaga, "High Sensitivity Fiber Laser Temperature Sensor," IEEE Sens. J. 15 (2015) 2399-2402.

[3] G. Liu, M. Han, and W. Hou, "High-resolution and fast-response fiber-optic temperature sensor using silicon Fabry-Perot cavity," Opt. Express 23 (2015) 7237-7247.

[4] B. Lee, S. B. Lee, Y. Rao, B. Sun, Y. Huang, J. Zhao, and Y. Wang, Ultra-sensitive temperature sensor based on liquid crystal infiltrated photonic crystal fibers, Proc. SPIE 9655 (2015) 96551W.

[5] S. j. Qiu, Y. Chen, F. Xu, "Temperature sensor based on an isopropanol-sealed photonic crystal fiber in-line interferometer with enhanced refractive index sensiti," Opt. Lett. 37 (2012) 863-865.

[6] T. Wu, Y. Liu, Z. Yu, H. Ye, W. Zhang, and H. He, "A nanometeric temperature sensor based on plasmonic waveguide with an ethanol-sealed rectangular cavity," Opt. Commun. 339 (2015) 1-6.

[7] Y. Peng, J. Hou, Z. Huang, and Q. Lu, Temperature sensor based on surface plasmon resonance within selectively coated photonic crystal fiber, Appl. Opt. 51 (2012) 6361-6367.

[8] T. Srivastava, R. Das, and R. Jha, "Highly Sensitive Plasmonic Temperature Sensor Based on Photonic Crystal Surface Plasmon Waveguide," Plasmon. 8 (2012) 515-521.

[9] X.-S. Lin and X.-G. Huan, "Tooth-shaped plasmonic waveguide filters with nanometeric sizes," Opt. Lett. 33 (2008) 2874-2876. 